\title{
VUV Emission Spectroscopy for Evaluation of Optical Thickness in He Cascade Arc Plasmas*)
}

\author{
Md. Anwarul ISLAM, Ryo SHIGESADA, Takumi YAMAGUCHI, Hayato KAWAZOME1), \\ Naoki TAMURA $^{2)}$, Hiroki OKUNO ${ }^{3)}$ and Shinichi NAMBA \\ Graduate School of Advanced Science and Engineering, Hiroshima University, Higashi-Hiroshima 739-8527, Japan \\ 1) National Institute of Technology, Kagawa College, Mitoyo 769-1192, Japan \\ ${ }^{2)}$ National Institute for Fusion Science, Toki 509-5292, Japan \\ ${ }^{3)}$ RIKEN, Nishina Center for Accelerator-Based Science, Wako 351-0198, Japan
}

(Received 16 November 2020 / Accepted 22 December 2020)

\begin{abstract}
We have generated a cascade arc plasma with a channel diameter of $8 \mathrm{~mm}$ for application to plasma window. Helium arc plasma up to a discharge current of $100 \mathrm{~A}$ were generated. We investigated the radiation trapping effect in the high-density He plasmas under various ambient gas pressures. In order to investigate the radiation trapping effect (optical thickness) in the high-density plasmas, we conducted vacuum UV (VUV) emission spectroscopy associated with He I resonance line. By observing the VUV spectrum attributed to He I resonance $\left({ }^{1} \mathrm{~S}-2{ }^{1} \mathrm{P}\right)$ transitions, we compared the emission intensities for different gas pressures. Lyman $\alpha$ intensity was increased by 3 times with a magnetic field due to well confinement of He plasma. The intensity decreased rapidly at high-ambient pressures due to the strong self-absorption. Therefore, the high-pressure arc plasma generated was classified into the optically thick one, and the self-absorption process of the resonance lines drastically influenced the population kinetics and energy transport.
\end{abstract}

(C) 2021 The Japan Society of Plasma Science and Nuclear Fusion Research

Keywords: cascade arc discharge, radiation trapping, VUV emission, radiative transfer equation, collisional radiative model, optically thick plasma, plasma window

DOI: $10.1585 /$ pfr.16.2406011

\section{Introduction}

Cascade arc discharge (wall-stabilized arc) that has intermediate floating electrodes is operated in high-gas pressure, by which high-density and high-temperature plasma can readily be generated $[1,2]$. The cascade arc discharge source expected an innovative virtual vacuum interface (plasma window) can separate vacuum from a high-gas pressure region without a large pumping system [3]. In addition, the plasma window has prospect as an alternative differential pumping system, splitting high- and lowpressure vacuum regions [4].

In optically thick plasmas, reabsorption of the emission occurs, and this phenomenon of absorption in the light sources itself is called as self-absorption (radiation trapping). The radiation trapping process significantly influences the population density of the excited levels. In general, for laboratory plasmas, only resonance lines are optically so thick that their spectral profile are drastically distorted due to the radiation trapping, especially at line center. If population densities do not suffer from the selfabsorption, then the intensity emitted from the plasma surface can be calculated as a function of spatiotemporal distributions of plasma temperature and density by using a

author's e-mail: d195402@hiroshima-u.ac.jp

*) This article is based on the presentation at the 29th International Toki Conference on Plasma and Fusion Research (ITC29). simple rate equation model or collisional-radiative model. The line intensity ratio of specific transitions provides a simple diagnostic tool to evaluate plasma parameters, such as electron temperature and density $[5,6]$. For the optically thick plasma, however, this method is no longer applicable, because the line intensity (population density) is significantly changed by the radiation trapping effect. In fact, the line center of the Lyman series has a dip structure in high-density plasma due to large absorption cross section around the central wavelength [7]. In the extreme case, we can find self-reversal line spectrum.

For accurate evaluation of plasma parameters by means of the line intensity ratio, the optical thicknesses both in plasma and along the line of sight to a detector have to be taken into account. Particularly, the reabsorption of the resonance lines due to ambient gas is of significance when the distance between light source and the detector is long. In order to evaluate the optical thickness in the highdensity $\mathrm{He}$ arc plasmas, we have measured the resonance line intensity (He I Lyman $\alpha$ line) and investigated the effect on the ambient gas pressure in the vacuum chamber with and without a magnetic field. The results obtained experimentally were compared with those calculated by using a collisional radiative (CR) model incorporated with radiation trapping effect [8]. 


\section{Experimental Setup}

Figures 1 (a) and (b) show a schematic diagram of the cascade arc discharge source and VUV emission spectroscopy, respectively. The arc device consisted of an anode, intermediate electrodes of ten plates, and a cathode. Tungsten material was employed for the anode because of its high melting point. For the cathode, a $\mathrm{LaB}_{6}$ disk with an opening of $5 \mathrm{~mm}$ was used. The water cooled floating intermediate electrodes of 5 plates with a thickness of $17 \mathrm{~mm}$ each with a channel opening of $8 \mathrm{~mm}$ in diameter, made of Mo were installed between the anode and cathode electrodes. Five intermediate electrodes had a tapered shape to confine the plasma into 8-mm discharge channel, as shown in Fig. 1 (a). The total length of the intermediate electrodes was $170 \mathrm{~mm}$. In order to avoid a spark discharge between the intermediate electrodes, teflon spacers with a thickness of $1 \mathrm{~mm}$ were installed between them. Helium gas was introduced through the cathode flange by a mass flow controller in a range of 0.05 to $0.5 \mathrm{~L} / \mathrm{min}$. The arc plasma was generated and expanded through the anode exit into a large vacuum chamber evacuated below $50 \mathrm{~Pa}$ by mechanical booster and rotary pumps. The discharge current and voltage were up to $100 \mathrm{~A}$ and $200 \mathrm{~V}$, respectively. Magnetic field was applied $\sim 80 \mathrm{mT}$, if necessary. The input powers at a 100-A discharge were 13.8 and $12.6 \mathrm{~kW}$ for with/without the magnetic field, respectively.

For characterization of the He plasma, emission spec-

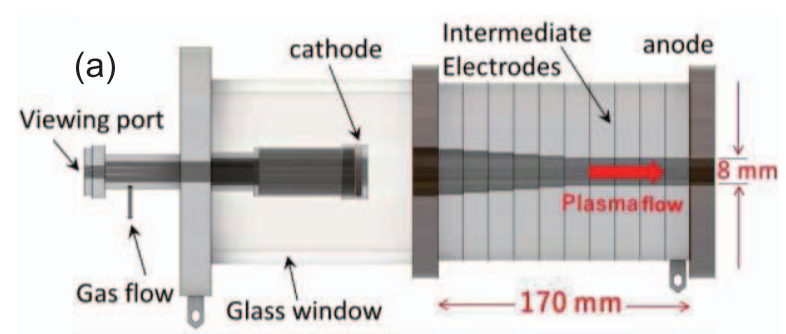

(b)

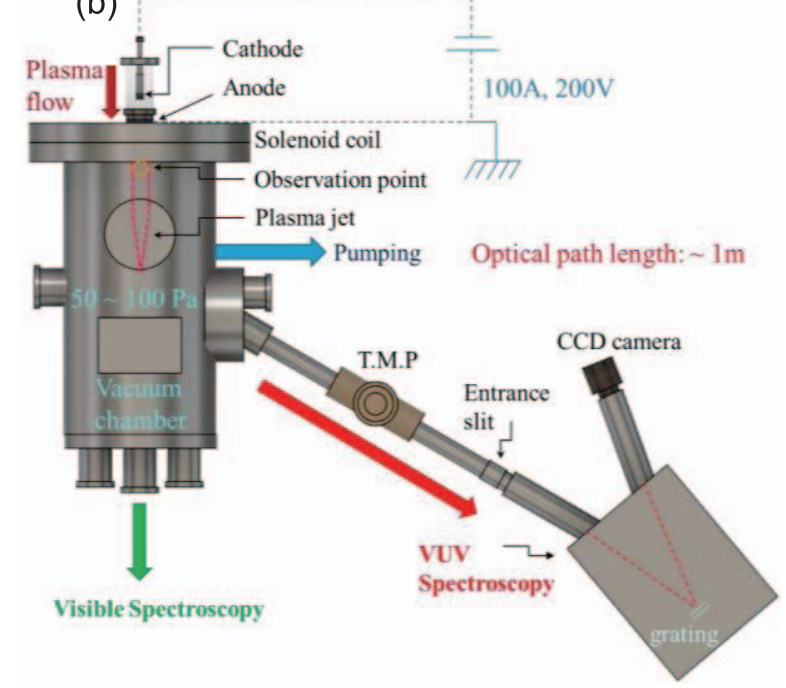

Fig. 1 (a) Cascade arc discharge source and (b) experimental setup for VUV emission spectroscopy. troscopy in VUV wavelengths was carried out. For the VUV emission, He I resonance spectra around the anode were observed by using a VUV spectrometer (Acton, VM$521, f=1 \mathrm{~m}, 1200$ grooves $/ \mathrm{mm}$ ), which was installed in the direction of $45^{\circ}$ with respect to the jet axis. The distance from the anode exit to the entrance slit was around $1 \mathrm{~m}$. In order to suppress the reabsorption in VUV optical path between the plasma emission region and the entrance slit of the spectrometer, we used a differential pump (turbo molecular pump), by which the pressure was kept to be as low as $1.27 \mathrm{~Pa}$. By changing the ambient gas pressure along VUV line of sight, we examined the effect of optical thickness of He I Lyman $\alpha$.

\section{Radiative Transfer Equation}

As described in the previous section, the selfabsorption for resonance lines cannot be neglected and rather controls the population kinetics of the excited states and even energy transport. Radiation trapping can be described by the radiative transfer equation [9],

$$
\mathrm{d} I=\varepsilon_{\mathrm{V}} \mathrm{d} x-\kappa_{\mathrm{v}} I \mathrm{~d} x,
$$

where $I$ is the intensity, $\kappa_{\mathrm{v}}$ is the absorption coefficient and $\varepsilon_{\mathrm{v}}$ is the emission coefficient. In the case of onedimensional homogeneous plasma, we obtain the following equation:

$$
I(x)=I_{0} e^{-\tau x}+\varepsilon_{\mathrm{v}} / \kappa_{\mathrm{v}}\left(1-e^{-\tau x}\right),
$$

where $\tau$ is the optical depth and is given by [10],

$$
\tau=\frac{\pi e^{2}}{M c} f_{i k}\left(1-\frac{n_{k}(r)}{n_{i}(r)} \frac{g_{i}}{g_{k}}\right) \lambda_{k i} \sqrt{\frac{M}{2 \pi k_{B} T}} \int_{0}^{x} n_{i}(r) \mathrm{d} r,
$$

where $f_{i k}$ is the absorption oscillator strength, $\lambda$ is the wavelength, $g$ is the statistical weight, $x$ is the boundary of particles, $M$ is the atomic mass, $T$ is the temperature of the particles, and $k_{B}$ is the Boltzmann constant. Here, $r$ is the spatial location, where the radiative transition from an upper state $k$ to a lower state $i$ occurs.

Figure 2 shows the Lyman $\alpha$ spectra for the optical thick and thin plasmas calculated by solving the radiative

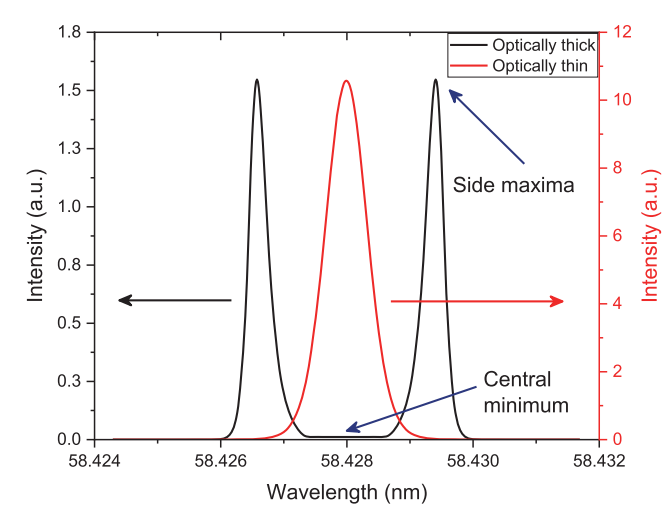

Fig. 2 He I Lyman $\alpha$ spectra for optical thick and thin plasmas. 
transfer equation. The gas temperature and density are $0.5 \mathrm{eV}$ and $6 \times 10^{17} \mathrm{~cm}^{-3}$, respectively. The initial spectral shape is assumed to be the Gaussian originating from the Doppler broadening. For the optically thin plasma, the profile still looks the Gaussian shape because of no selfabsorption in plasma. However, for the optically thick $(\tau \gg 1)$, the Lyman $\alpha$ peak intensity is drastically decreased by $\sim 10^{6}$, and the profile is radically deformed, resulting in a dip structure at line center as indicated by "central minimum" in the figure. Consequently, only wing components ("side maxima") can be observed.

\section{Results and Discussion}

Figure 3 (a) plots the VUV spectra of He I resonance lines $\left(1{ }^{1} \mathrm{~S}-n p{ }^{1} \mathrm{P}\right)$ at a discharge current of $100 \mathrm{~A}$ and a gas flow rate of $0.05 \mathrm{~L} / \mathrm{min}$. The magnetic field was not applied. Line emissions attributed to principal quantum numbers of $n=2(\lambda=58.4 \mathrm{~nm})$ and $n=3(53.7 \mathrm{~nm})$ are observed in this spectral window. The resonance intensities are increased with decreasing discharge gas pressure, as shown in Fig. 4.

Figure 3 (b) shows the VUV spectra of He I resonance lines for $0.05 \mathrm{~L} / \mathrm{min}$ and $100 \mathrm{~A}$ discharge with the magnetic field. Compared with the case (a), many lines attributed $\mathrm{O}$ II, B II and B III ions appear in (b). The result implies that under weak magnetic field the cathode material, $\mathrm{LaB}_{6}$, is significantly sputtered by the ion bombardment. Magnetic field confinement of plasma changes the emission behaviors and constituents in the plasma. The result of this drastic variation in emissions is unclear because of the reasons of appearing many impurity lines with the magnetic field are unknown. Further experiment is needed for the precise clarification. Without the magnetic field, the charged particles readily interact with the channel wall, resulting in the heat loss onto the wall. Consequently, the plasma jet has a lower temperature at the anode exit and lowers the Lyman $\alpha$ intensity, as shown in Fig. 4. In fact, the Lyman $\alpha$ intensity was increased by $\sim 3$ times under a discharge gas pressure of $1.3 \mathrm{kPa}$ and application of the magnetic field.

The self-absorption causes the intensity of He I Lyman $\alpha$ being decreased. Note that the length between the anode exit and the entrance slit of VUV spectrometer was around $1 \mathrm{~m}$. For a homogeneous ambient pressure, the reabsorption of Lyman $\alpha$ due to neutral gas is expressed by $I_{0} \exp \left(-\kappa_{v} x\right)$, as shown in Eq. (2). This equation can be rewritten by $I_{0} \exp (-\sigma n x)$, where $\sigma$ is the absorption cross section and $n$ is the atomic density. In order to evaluate the effect of the self-absorption due to ambient gas in the vacuum chamber, therefore, we changed the pressure (absorber density) along the line of sight and then measured the Lyman $\alpha$ intensity. Figure 5 shows the dependence of the Lyman $\alpha$ intensity on the ambient gas pressure under the magnetic field application. The spectra without the field show similar trend with those with the field. The lower gas pressure shows the increase in intensity and the
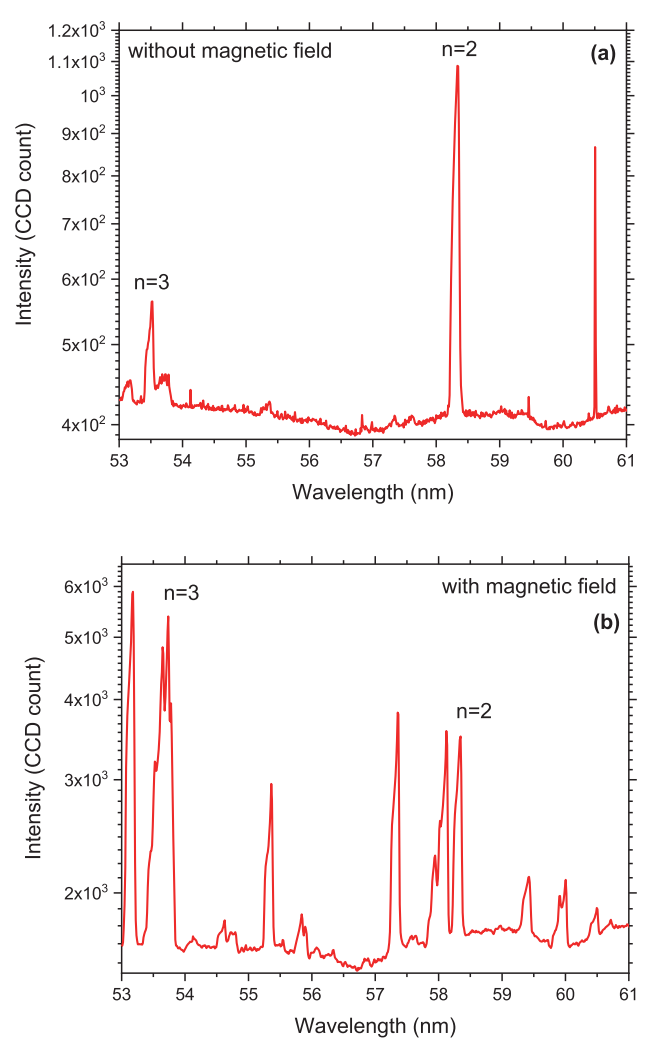

Fig. 3 VUV spectra of $\mathrm{He}$ I for $0.05 \mathrm{~L} / \mathrm{min}$ and 100 A discharge, (a) without and (b) with a magnetic field of $50 \mathrm{G}$.

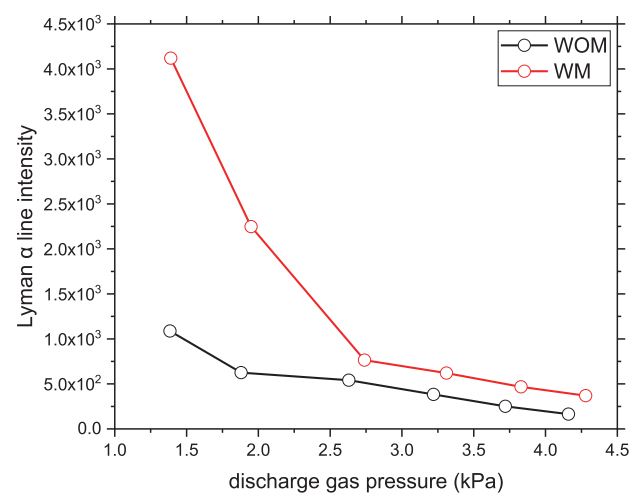

Fig. 4 Spectral intensity of He I Lyman $\alpha$ intensity against the discharge gas pressure.

intensity decreases exponentially, which follows the above equation.

The simple absorption ratio evaluated by using the equation of $I / I_{0}=\exp (-\sigma n x)$ is also shown in Fig. 5. Here, the absorption cross section $(\sigma)$ is $3.28 \times 10^{-7} \lambda^{2} \mathrm{~cm}^{2}$ and $x$ is $290 \mathrm{~mm}$ (sight line length), and the atomic density $n$ was estimated by using the equation of state, $\mathrm{P}=[n k T]$ ( $T=300 \mathrm{~K}$, room temperature). The changes of the absorption ratio follow the trend observed in the experimental results. 


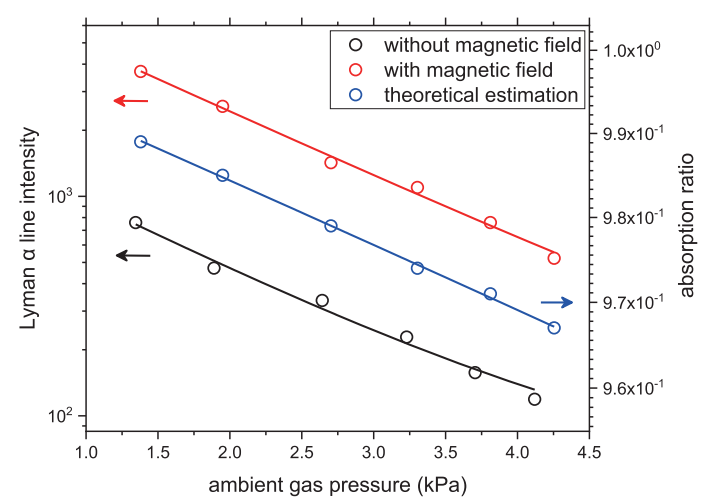

Fig. 5 Lyman $\alpha$ intensity against ambient gas pressure with and without the magnetic field and the theoretical evaluation of Lyman $\alpha$ intensity.

\section{Summary}

We have developed an $8 \mathrm{~mm}$ diameter cascade arc source for differential pumping plasma window. We observed the VUV spectrum attributed to He I resonance lines. We investigated the effect of radiation trapping on high-pressure He arc plasma. The optical thickness significantly influenced the emission intensity of resonance lines in the optically thick plasma for high-ambient pressures.

[1] S. Namba, T. Endo, S. Fujino, C. Suzuki and N. Tamura, Rev. Sci. Instrum. 87, 083503 (2016)

[2] S. Namba et al., Phys. Plasmas 25, 113511 (2018).

[3] A. Hershcovitch, J. Appl. Phys. 78, 5283 (1995).

[4] H. Imao et al., Phys. Rev. Accel. Beams 15, 123501 (2012).

[5] M. Ivkovié, S. Jovićević and N. Konjević, Spectrochim. Acta B 59, 591 (2004).

[6] Md. Anwarul Islam, T. Yamaguchi, K. Fukuyama, H. Kawazome, N. Tamura and S. Namba, IEEE Trans. Plasma Sci. Special Issue (2020).

[7] R.D. Cowan and G.H. Dieke, Rev. Mod. Phys. 20, 418 (1948).

[8] X. Yuan and L. Raja, IEEE Trans. Plasma Sci. 31, 495 (2003).

[9] H. Zwicker, Evaluation of plasma parameters in optically thick plasmas. Plasma Diagnostics, 1968.

[10] S. Namba, M. Goto, H. Tsuboi, T. Oda and K. Sato, J. Appl. Phys. 88, 3182 (2000). 